\title{
Rheumatic fever: the rebound phenomenon returns
}

Joshua Osowicki ${ }^{1,2,3,4}$

Jeremy P. Carr ${ }^{3}$

Andrew C. Steer ${ }^{1,2,3}$

1. Group A Streptococcus Research Group, Murdoch Children's Research Institute, Melbourne, Victoria, Australia

2. Department of Paediatrics, University of Melbourne, Melbourne, Victoria, Australia

3. Infectious Diseases Unit, Department of General Medicine, The Royal Children's Hospital Melbourne, Victoria, Australia

4. Department of Paediatric Infection and Immunity, Monash Children's Hospital, Clayton, Australia

Running header: Rheumatic fever rebound phenomenon

Address for correspondence: Joshua Osowicki

Group A Steptococcus Research Group

Murdoch Children's Research Institute

50 Flemington $\mathrm{Rd}$

Parkville, VIC

Australia, 3052

joshua.osowicki@rch.org.au

Competing interests: all authors report they have no financial or other competing interests to report in relation to this submission

This is the author manuscript accepted for publication and has undergone full peer review but has not been through the copyediting, typesetting, pagination and proofreading process, which may lead to differences between this version and the Version of Record. Please cite this article as doi: $10.1111 /$ jpc.13848

This article is protected by copyright. All rights reserved. 


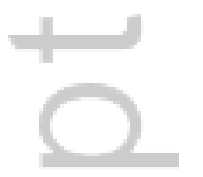




\section{Background}

The global burden of acute rheumatic fever (ARF) and subsequent rheumatic heart disease (RHD) remains high. By conservative estimates there are more than 300,000 incident cases of ARF annually and more than 30 million prevalent cases of RHD, responsible for at least 300,000 deaths every year. ARF and RHD have become uncommon in high-income countries, outside of specific sub-groups including indigenous peoples. ${ }^{1}$ Accurate and timely diagnosis of ARF remains important in preventing RHD and its complications including heart failure and stroke. ${ }^{2-6}$ Diagnosis and management of ARF can be difficult and atypical clinical manifestations or patterns may be misinterpreted, especially in settings where it is rarely seen. We present an unusual case which also highlights divergent approaches to anti-inflammatory therapy in ARF.

\section{Case report}

A nine-year-old boy of Samoan descent presented with a one-week history of fever and migratory arthralgia affecting his knees, ankles and spine. Two weeks prior, he had been treated for microbiologically-confirmed Streptococcus pyogenes impetigo. There was no history of recent pharyngitis. His mother recalled a similar episode of fever and joint pain two years prior, which resolved without medical assessment. His older brother had previously been diagnosed with ARF. Cardiac examination revealed a grade 2 systolic and early diastolic murmur, loudest at the upper left sternal edge, without clinical signs of heart failure or infective endocarditis. No arthritis, rash or subcutaneous nodules were present. Electrocardiogram demonstrated first-degree heart block (PR interval $210 \mathrm{~m} / \mathrm{s}$, upper limit of normal for age $160 \mathrm{~m} / \mathrm{s}$ ). The C-reactive protein (CRP) was $82 \mathrm{mg} / \mathrm{L}$ and erythrocyte sedimentation rate (ESR) $40 \mathrm{~mm} / \mathrm{hr}$. S. pyogenes was not isolated by throat swab culture. The antistreptolysin $\mathrm{O}$ antibody titre was $2260 \mathrm{IU} / \mathrm{mL}$ (upper limit of normal for age <276). Echocardiographic findings were consistent with mitral and aortic rheumatic valvulitis. (Figure 1)

He was commenced on ibuprofen (400mg three times a day) and the arthralgia resolved within 72 hours. He was discharged soon after without any further regular anti-inflammatory therapy, with a plan 
for continuing secondary prophylaxis for RHD with intramuscular benzathine penicillin G.

He presented to our tertiary paediatric hospital two weeks later with recrudescence of fever, lower limb and back pain, worse than before, restricting him to bed. Arthritis was now clinically evident in the left hip, right ankle and right midfoot. CRP was $207 \mathrm{mg} / \mathrm{L}$ and ESR $132 \mathrm{~mm} / \mathrm{hr}$. Cardiac findings were unchanged. Treatment with naproxen $(10 \mathrm{mg} / \mathrm{kg}$ twice daily) led to rapid improvement and he was discharged after two days, when able to mobilize. Arthritis resolved over several days and naproxen was ceased after four weeks, only for arthritis to return two weeks later, affecting the shoulder and knee. Again, naproxen $(10 \mathrm{mg} / \mathrm{kg}$ twice daily) was administered, with good effect. Arthralgia returned when the dose was halved after two weeks $(5 \mathrm{mg} / \mathrm{kg}$ twice daily) and disappeared once more at the higher dose. Anti-inflammatory medications were finally ceased four months after the initial diagnosis, without further relapse.

Throughout this period, he had no cardiac symptoms or signs of heart failure, and echocardiographic findings were unchanged. Follow-up echocardiography ten months later showed improved aortic regurgitation and no residual mitral regurgitation.

\section{Discussion}

This case highlights the rebound phenomenon in ARF, a clinical syndrome of prolonged inflammation characterised by the recrudescence of previously suppressed clinical and laboratory evidence of rheumatic activity upon withdrawal or reduction of anti-inflammatory therapy. Rebound is a separate entity from recurrent ARF and does not increase the risk of progression to chronic RHD. Historically, when treatment with salicylates and/or corticosteroids was very common, significant clinical evidence of rebound occurred in approximately $15 \%$ of ARF cases, and was more common when steroids were given. ${ }^{7}$ It may occur less frequently now because of narrower indications for anti-inflammatory therapy. 
Anti-inflammatory therapy has a long history in ARF. The symptomatic response to aspirin for arthritis in ARF is so impressive that a poor response should lead to reconsideration of the diagnosis. ${ }^{2}$ However, anti-inflammatory treatment does not alter progression to RHD. ${ }^{8}$ Contemporary recommendations for anti-inflammatory therapy differ across guidelines (Table 1). The only universal routine indication is for symptomatic relief of joint symptoms. Traditional standard treatment has been with aspirin, but newer non-steroidal anti-inflammatory drugs (NSAID) such as naproxen may be equally effective, more convenient, and safer. ${ }^{9}$. Evidence for use of corticosteroids is not strong, and weaker still for intravenous immunoglobulin, and recommendations based on expert opinion limit their use to severe cases of carditis and chorea. ${ }^{8}$

It has previously been suggested that the average combined clinical and pathological natural history of an untreated ARF episode is three months, up to six months with severe carditis, and in less than five percent of cases longer than six months. ${ }^{10,11}$ Anti-inflammatory treatment suppresses inflammation but "does not terminate the active process" and is counter-intuitively associated with a longer total duration of inflammation. Rebound symptoms occur when treatment is stopped "if the attack has not yet run its course." ${ }^{10}$ A 1961 retrospective single-centre cohort study of 265 consecutive children and adolescents with ARF specifically examined this rebound phenomenon. The study recorded clinical features, details of anti-inflammatory treatment, and closely monitored biochemical inflammatory markers ${ }^{7,12}$ Rebound episodes usually occurred within one to two weeks of stopping antiinflammatory therapy. Significant clinical rebound episodes affected 41/231 patients treated with antiinflammatory drugs (17.7\%), and were characterised by fever, elevated inflammatory markers, and any one or more of: tachycardia, joint pain, pathological cardiac auscultatory findings, congestive cardiac failure, subcutaneous nodules, and erythema marginatum. Some episodes were "worse than the manifestations of the original attack". Isolated laboratory evidence of rebound rheumatic activity (CRP, ESR) occurred in 84/231 (36\%) of all patients treated with anti-inflammatory drugs and subsided spontaneously in all cases. Clinical rebounds were more common with steroid treatment compared to aspirin, with an acknowledged risk of confounding by indication. Rebound episodes did 
not occur in patients who did not receive anti-inflammatory treatment, in those who received at least eight weeks of anti-inflammatory therapy, with aspirin or else steroids initially followed by “overlapping" aspirin.

Most clinical rebound episodes resolved in one week without further treatment, and rarely continued for longer than two weeks. Clinical evidence of carditis did not occur during rebound episodes in patients for whom carditis was not part of their initial presentation. In 10/35 patients with carditis at presentation, new pathological cardiac auscultatory findings were recorded during rebound episodes. Without the benefit of echocardiographic correlation and prior to recognition that anti-inflammatory therapy does not prevent progression to RHD, the authors cautiously concluded that clinical rebound in patients with carditis might carry the "threat of additional cardiac damage." They recommended reserving further anti-inflammatory therapy for rebound episodes associated with pericarditis, increasing cardiomegaly, or cardiac failure, and highlighted the likelihood of further rebound episodes if anti-inflammatory therapy was reinstituted.

In retrospect, the initial ARF rebound episode for the nine-year-old boy discussed here was a signifier for prolonged and pronounced inflammation and, once restarted, anti-inflammatory therapy should have been continued and very slowly weaned over eight to twelve weeks. With the declining incidence of ARF in industrialised countries, an atypical clinical course of the disease may be misinterpreted. It is important to distinguish rebound from recurrent ARF which carries a risk of progressive RHD.

Recurrent ARF usually occurs at least eight weeks after the previous episode, following an intervening streptococcal infection. Awareness of rebound can also prevent unnecessary investigations and additional treatment for conditions such as infective endocarditis, septic arthritis, and juvenile idiopathic arthritis, which may present similarly. Amongst patients who are treated with antiinflammatory drugs, most require only short courses. Symptomatic rebound carditis is rarely present during rebound episodes, and other signs or laboratory features of rebound do not mandate echocardiographic re-evaluation. For the minority who do experience clinical rebound with joint pain 
after stopping anti-inflammatory treatment, it should be reinstituted and continue at least until resolution of symptoms and laboratory markers of inflammation return to normal.

This article is protected by copyright. All rights reserved. 


\section{Learning points}

- Acute rheumatic fever (ARF) and rheumatic heart disease (RHD) now occur rarely in highincome countries, but they remain a major cause of infection-related morbidity and mortality worldwide.

- Not all cases of ARF require anti-inflammatory therapy (e.g. aspirin or naproxen, prednisolone). The only universal recommendation is for relief of joint symptoms.

- Whenever anti-inflammatory therapy is administered there is a risk of rebound clinical and laboratory features of ARF when treatment is stopped or reduced.

- This 'rebound phenomenon' is distinct from recurrent ARF. Unlike recurrent ARF, rebound episodes are not associated with increased risk of progressive RHD.

This article is protected by copyright. All rights reserved. 


\section{Question 1.}

It is your first day on the cardiology ward and you are caring for an adolescent who has just had a mitral valvuloplasty for rheumatic heart disease. You are surprised to discover this was her first admission for ARF or RHD and that she can't ever remember having had an episode of pharyngitis. She was treated last year for 'culture-negative septic arthritis' of her left knee with a prolonged course of intravenous then oral antibiotics. You can see old skin sores on her legs, and her mother tells you she has been treated many times for scabies. What is going on?
A. Kingella kingae causes septic arthritis in children, and endocarditis. It is difficult to identify with conventional culture methods. $K$, kingae is a more likely explanation than RHD.
B. This is not an unusual presentation of RHD. You should not be surprised.
C. This is a classic case of eosinophilic myocarditis due to chronic scabies infection.
D. Adolescents are notoriously unreliable historians. She probably just does not remember when she had pharyngitis.

E. Do an electrocardiogram. If the PR interval is normal, this isn't RHD and a congenital mitral valve abnormality is the most likely reason she needed surgery.

\section{Answer: B}

A history of pharyngitis is unusual in indigenous Australians with ARF, whereas skin infections are common. This has led many experts to question the long-held belief that only GAS pharyngitis can lead to ARF. Many, and perhaps most, people with RHD were never diagnosed with ARF, which is one of the reasons that a GAS vaccine is such an attractive idea. There are now global consensus guidelines for the echocardiographic diagnosis of RHD, which is associated with characteristic abnormalities, typically of the mitral and/or aortic valve(s). Monoarthritis is a well-recognised presentation of ARF and is considered a major diagnostic feature in moderate-to-high-risk populations. However, in all settings it is important to consider the long differential diagnosis for monoarthritis. Scabies is a very important risk factor for recurrent GAS skin infections, as has become apparent from 
the impressive reduction in impetigo associated with population-wide scabies treatment, such as with mass-drug administration of ivermectin. Scabies does not cause myocarditis.

\section{Question 2.}

You admit a 15-year-old boy with polyarthralgia, fever, elevated ESR and CRP. He has no cough, coryza, rash, or conjunctivitis, although he did have a sore throat a couple of weeks ago. Three months earlier he was admitted to your hospital with monoarthritis and diagnosed with ARF. He had 10 days of anti-inflammatory treatment. Could this be a case of the ARF rebound phenomenon?
A. If he was treated with corticosteroids during the first admission, this is likely to be a rebound episode.
B. Yes, if GAS is not identified by throat swab culture, and the ASOT is negative, then this is the rebound phenomenon.
C. No. This could be a recurrent ARF episode due to poor adherence to secondary prophylaxis with intramuscular benzathine penicillin.
D. It depends on the findings of an echocardiogram.
E. No. This is a case of recurrence due to penicillin-resistant GAS. His secondary prophylaxis should be switched to azithromycin.

\section{Answer: C}

The diagnosis of recurrent ARF can be challenging and requires a high index of suspicion. Recurrent ARF can be diagnosed without fulfilling the Jones diagnostic criteria. A throat swab, ASOT, and repeat echocardiogram may be helpful in characterising the episode and its impact, but none will prove the current episode is due to the rebound phenomenon. Although the natural history of ARF may be as long as three months, in this case a very long asymptomatic period has passed since the initial ARF episode and cessation of anti-inflammatory treatment. Poor adherence to painful intramuscular benzathine penicillin injection given as secondary prophylaxis is considered a major problem, but the 
evidence for oral alternatives is not strong. Penicillin-resistant GAS has never been reported. It also sounds as if there might have been a preceding episode of GAS pharyngitis.

\section{Question 3:}

You are doing a locum in remote northern Australia. You see a child with unusual movements in the emergency department and suspect she may have Sydenham chorea.
A. Start intravenous benzylpenicillin (penicillin $\mathrm{G}$ ) immediately, and urgently transfer to a tertiary centre.
B. Prescribe a course of oral phenoxymethylpenicillin (penicillin V) and review in seven days.
C. Start naproxen. If her symptoms do not improve, switch to prednisolone.
D. Arrange hospital admission for evaluation, including an echocardiogram.
E. Start sodium valproate.

\section{Answer: D}

Chorea is a major diagnostic criterion for ARF and in high-risk settings when differential diagnoses have been excluded the movement disorder is considered sufficient to make the diagnosis of ARF even in the absence of other features. An early echocardiogram is very important to assess for evidence of rheumatic heart disease. Steroids (sometimes intravenous immunoglobulin) and anti-consultants are used for severe cases. There is no role for intravenous antibiotics in ARF. Oral penicillin V is an alternative for secondary prophylaxis, but the evidence supporting intramuscular benzathine penicillin every 21-28 days is stronger and it is the preferred choice. 


\section{References}

- $\quad$ 1. Carapetis JR, Beaton A, Cunningham MW, et al. Acute rheumatic fever and rheumatic heart disease. Nat Rev Dis Primers. 2016; 2: 15084.

- $\quad$ 2. RHDAustralia (ARF/RHD writing group), National Heart Foundation of Australia and the Cardiac Society of Australia and New Zealand.

- $\quad$ Australian guideline for prevention, diagnosis and management of acute rheumatic fever and rheumatic heart disease (2nd edition). 2012.

- $\quad$ 3. Heart Foundation of New Zealand. New Zealand Guidelines for Rheumatic Fever: Diagnosis, Management and Secondary Prevention of Acute Rheumatic Fever and Rheumatic Heart Disease: 2014 Update. Available at: www.heartfoundation.org.nz

4. World Health Organization. Pocket book of hospital care for children: guidelines for the management of common childhood illnesses - 2nd ed. 2013.

- $\quad$ 5. Working Group on Pediatric Acute Rheumatic F, Cardiology Chapter of Indian Academy of $\mathrm{P}$, Saxena A, et al. Consensus guidelines on pediatric acute rheumatic fever and rheumatic heart disease. Indian Pediatr. 2008; 45: 565-573.

- $\quad$ 6. Sociedade Brasileira de C. [Brazilian guidelines for the diagnosis, treatment and prevention of rheumatic fever]. Arq Bras Cardiol. 2009; 93: 3-18.

- $\quad$ 7. Feinstein AR, Spagnuolo M, Gill FA. The rebound phenomenon in acute rheumatic fever. I. Incidence and significance. Yale J Biol Med. 1961; 33: 259-278.

- $\quad$ 8. Cilliers A, Adler AJ, Saloojee H. Anti-inflammatory treatment for carditis in acute rheumatic fever. Cochrane Database Syst Rev. 2015: CD003176.

- $\quad$ 9. Hashkes PJ, Tauber T, Somekh E, et al. Naproxen as an alternative to aspirin for the treatment of arthritis of rheumatic fever: a randomized trial. J Pediatr. 2003; 143: 399-401.

- $\quad$ 10. Markowitz M, Gordis L. Rheumatic fever. Major Probl Clin Pediatr. 1972; 11: 1-309.

- $\quad$ 11. Taranta A, Spagnuolo M, Feinstein AR. "Chronic" rheumatic fever. Ann Intern Med. 1962; 56: 367-388.

- $\quad$ 12. Spagnuolo M, Feinstein AR. II. Treatment and Prevention. Yale J Biol Med. 1961; 33: 279-298. 
Table 1. Guideline recommendations for anti-inflammatory therapy of acute rheumatic fever

\begin{tabular}{|c|c|c|c|}
\hline Guideline & Indications & Drug(s) & Duration \\
\hline \multirow{2}{*}{$\begin{array}{l}\text { World Health } \\
\text { Organization }^{4}\end{array}$} & all patients & aspirin & 4-8 weeks \\
\hline & heart failure & steroids & 1 week \\
\hline \multirow[t]{2}{*}{ Brazil $^{6}$} & arthritis & $\begin{array}{l}\text { aspirin (first line) } \\
\text { naproxen }\end{array}$ & 4 weeks \\
\hline & moderate-severe carditis & steroids & 12 weeks \\
\hline \multirow[t]{2}{*}{ India $^{5}$} & arthritis $+/$ - mild carditis & $\begin{array}{l}\text { Aspirin (first line) } \\
\text { naproxen (aspirin intolerance) } \\
\text { steroids }\end{array}$ & $\begin{array}{l}\text { "Must be" } 12 \text { weeks, and switch } \\
\text { to steroids if no response in } 4 \\
\text { days }\end{array}$ \\
\hline & moderate-severe carditis & steroids & $\begin{array}{l}\text { Until ESR normal, usually } 2 \\
\text { weeks, taper and overlap aspirin } \\
\text { to complete total } 12 \text { weeks }\end{array}$ \\
\hline \multirow[t]{3}{*}{$\begin{array}{l}\text { Australia }^{2} \text { and } \\
\text { New Zealand } \\
\text { New }^{3}\end{array}$} & arthritis, severe arthralgia & $\begin{array}{l}\text { aspirin (not recommended - New Zealand) } \\
\text { naproxen (first line - New Zealand) } \\
\text { other NSAIDs }\end{array}$ & $\begin{array}{l}\text { Usually } 1-2 \text { weeks, longer courses } \\
\text { for persistent/rebound symptoms } \\
\text { and elevated CRP/ESR (6-12 } \\
\text { weeks, rarely } \geq 6 \text { months) }\end{array}$ \\
\hline & severe carditis & consider steroids & $\begin{array}{l}\text { Days }-3 \text { weeks, with overlapping } \\
\text { aspirin to avoid rebound after } \\
\text { stopping steroids }\end{array}$ \\
\hline & severe chorea & $\begin{array}{l}\text { consider steroids and IVIG only for severe } \\
\text { refractory cases }\end{array}$ & No recommendation \\
\hline
\end{tabular}

NSAID: non-steroidal anti-inflammatory drug; ESR: erythrocyte sedimentation rate; CRP: Creactive protein IVIG: intravenous immunoglobulin 
Figure 1. Clinical course

This article is protected by copyright. All rights reserved. 


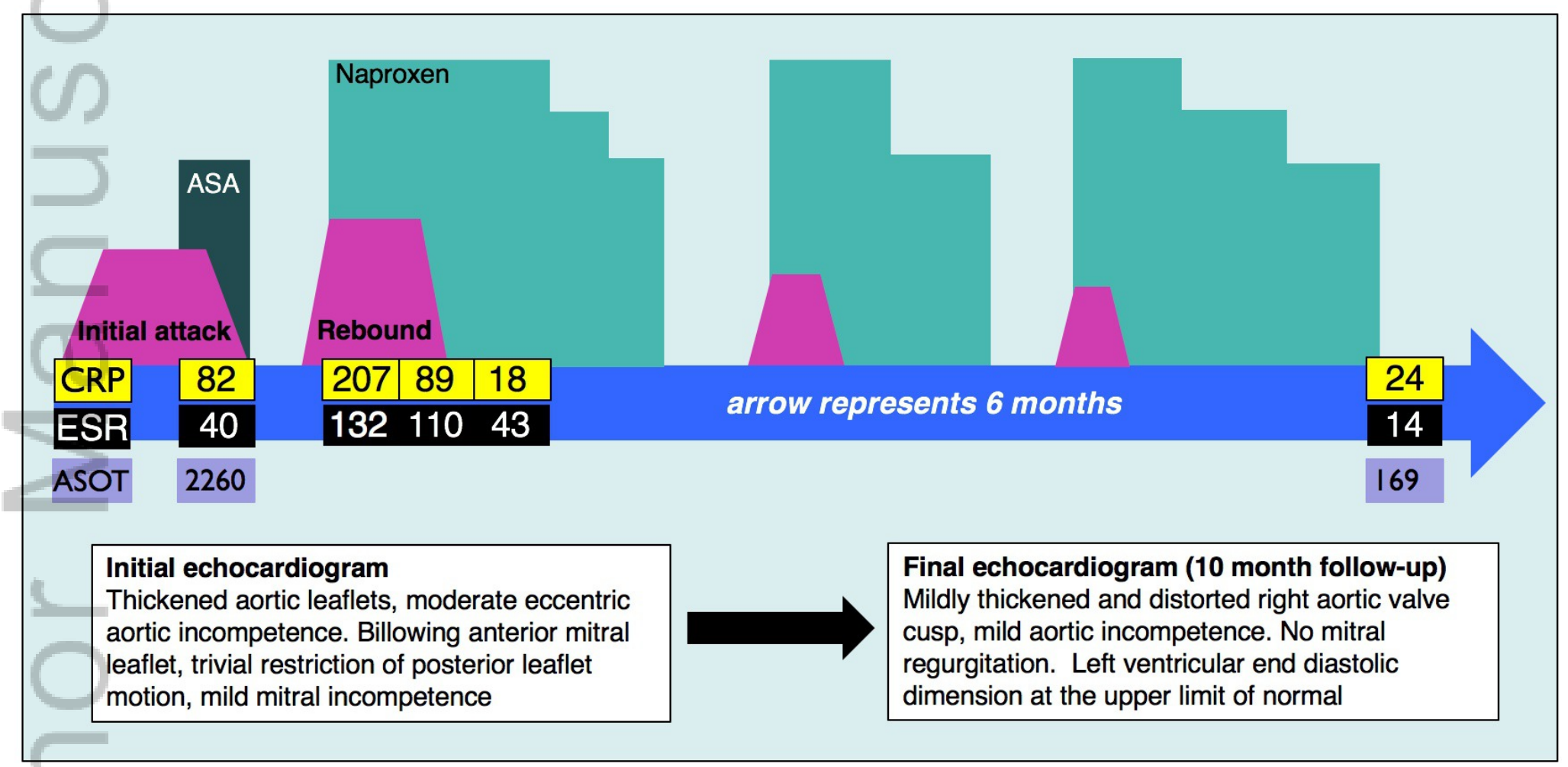

JPC_13848_Figure 1.jpeg

This article is protected by copyright. All rights reserved. 


\section{University Library}

\section{- M M I E R R A A gateway to Melbourne's research publications}

Minerva Access is the Institutional Repository of The University of Melbourne

Author/s:

Osowicki, J;Carr, JP;Steer, AC

Title:

Rheumatic fever: The rebound phenomenon returns

Date:

2018-06-01

Citation:

Osowicki, J., Carr, J. P. \& Steer, A. C. (2018). Rheumatic fever: The rebound phenomenon returns. JOURNAL OF PAEDIATRICS AND CHILD HEALTH, 54 (6), pp.685-688. https:// doi.org/10.1111/jpc.13848.

Persistent Link:

http://hdl.handle.net/11343/283604 